\title{
Elucidation of Regional Mechanical Properties of Brain Tissues Based on Cell Density
}

\author{
Chi Zhang, Long Qian, Hongwei Zhao* \\ School of Mechanical and Aerospace Engineering, Jilin University, Changchun 130025, China
}

\begin{abstract}
Research on the mechanical properties of brain tissue has received extensive attention. However, most of the current studies have been conducted at the phenomenological level. In this study, the indentation method was used to explore the difference in local mechanical properties among different regions of the porcine cerebral cortex. Further, hematoxylin-eosin and immunofluorescence staining methods were used to determine the correlation between the cellular density at different test points and mechanical properties of the porcine cerebral cortex. The frontal lobe exhibited the strongest viscosity. The temporal lobe displayed the lowest sensitivity to changes in the indentation speed, and the occipital lobe exhibited the highest shear modulus. Additionally, the shear modulus of different areas of the cerebral cortex was negatively correlated with the total number of local cells per unit area and positively correlated with the number of neuronal cell bodies per unit area. Exploration of the mechanical properties of the local brain tissue can provide basic data for the establishment of a finite element model of the brain and mechanical referential information for the implantation position of brain chips.
\end{abstract}

Keywords: cerebral cortex, mechanical properties, staining, correlation, cellular density

Copyright (C) The author(s) 2021.

\section{Introduction}

During the long course of human life, numerous brain diseases, such as traumatic brain injury, epilepsy, and tumours, can occur. These diseases pose severe threat to human life. For improving the treatment of brain diseases, many aspects based on numerous disciplines, such as cytology ${ }^{[1]}$ and neurology ${ }^{[2]}$, have been explored. Recent studies have shown that mechanical signals act as a pivotal messenger during the development of biological systems, in the interpretation of brain pathology ${ }^{[3]}$, and for the treatment of major diseases ${ }^{[4]}$. Therefore, Capturing brain mechanical signals and exploring the mechanical properties of brain tissue became a hot interdisciplinary research topic and gradually combined mechanobiology, biology, and medicinal science. With the deepening of research and further development, many methods such as tension ${ }^{[5]}$, shearing $^{[6]}$, compression $^{[7]}$, and indentation ${ }^{[8]}$ for testing the mechanical properties of brain tissues have been developed. Because of the selective expression of genes, the brain structure is complex and the mechanical properties of different parts of the brain vary. Numerous experiments have identified significant differences in the mechanical properties of the cerebral cortex, cerebellum, pons, and medulla oblongata ${ }^{[9,10]}$. Nevertheless, the results vary widely depending on the sample age ${ }^{[11]}$, time of death ${ }^{[12]}$, and test area ${ }^{[13]}$. Even for the same brain part, test results differ considerably. In summary, regarding the mechanical properties of brain tissues, researchers have only explored the tip of the iceberg.

In terms of characterising the mechanical properties of biological tissues, the indentation method is highly flexible and accurate because it is not limited to the shape of the sample and does not depend on the location of the test. It can be used for local testing under complex conditions. Recently, indentation method was applied to characterise the mechanical properties of the human brain $^{[14]}$ and brain tissues of rats ${ }^{[15]}$, pigs ${ }^{[16]}$, and cattle ${ }^{[12]}$. However, the current research is based more on phenomenology and does not link the macroscopic mechanical phenomena with the microstructure or explore the correlation between them. Previous studies have shown that mechanical signals play an important role in brain growth and development as well as in the treatment and control of brain diseases. For instance, neurons and

\footnotetext{
*Corresponding author: Hongwei Zhao

E-mail: hwzhao@jlu.edu.cn
} 
astrocytes can activate protein channels through unique mechanical sensitivity ${ }^{[17]}$. Neurons can sense and adapt to mechanical signals in the environment and exhibit different growth rates depending on the available substrate $^{[18]}$. Therefore, the combination of mechanical properties and microstructure of brain tissue holds great significance in improving the understanding of brain development and optimising the treatment of brain diseases. A recent study reported that the stiffness of the mouse spinal cord correlates strongly with the areas of cell nuclei and cellular in-plane proximity ${ }^{[19]}$. Budday et al. revealed a negative correlation between cell count and stiffness ${ }^{[20]}$. These reports indicate that the number and density of cells are closely related to the mechanical properties of tissues. In addition, in a study by Budday et $a l$, because of the limitation of the staining method, neurons and glial cells could not be distinguished ${ }^{[20]}$. Therefore, the correlation between the stiffness of brain tissues and a single type of cell remains to be explored.

In this study, we aimed to construct a bridge between medicine and mechanics. The mechanical properties of the porcine brain tissue in different regions were characterised through large deformation indentation. Subsequently, hematoxylin and eosin (H \& E) staining was used to count the total number of nuclei per unit area. Meanwhile, immunofluorescence staining was used to observe neurons, and the neuronal cell bodies per unit area were counted under a laser confocal microscope. The purpose of this study was to explore the correlation between local mechanical properties and cell distribution in the cerebral cortex by combining mechanical properties and microstructure. The study provided basic data for the establishment of a finite element model of the brain and referential data for future brain chip implantation position.

\section{Materials and methods}

\subsection{Sample preparation}

Because of the similarity between the porcine and human brains in terms of the anatomy, myelination, gyrification, development, and quantifiable behaviour traits, porcine brain is considered a common substitute to the human brain for conducting research ${ }^{[21]}$, particularly in the field of neuroscience ${ }^{[22,23]}$. Previous studies have shown that the mechanical properties of the porcine and human brains are highly similar in vitro ${ }^{[24]}$. In addition, the porcine brain tissue was chosen in the present study because of the availability and possibility of minimising the post-mortem time during testing ${ }^{[25]}$. The porcine brains used in this experiment were obtained from approximately 5-6-month-old adult pigs $(n=27)$ from a local slaughterhouse. Fresh porcine brains were removed within $30 \mathrm{~min}$ of decapitation. To ensure the best storage environment, the isolated tissues were stored and transferred in artificial cerebrospinal fluid (aCSF) containing $124 \mathrm{mM} \mathrm{NaCl}, 26 \mathrm{mM} \mathrm{NaHCO}$, $2.5 \mathrm{mM} \mathrm{KCl}$, $2 \mathrm{mM} \mathrm{CaCl}_{2}, 1.25 \mathrm{mM} \mathrm{NaH}_{2} \mathrm{PO}_{4}, 1 \mathrm{mM} \mathrm{MgCl}$, and $10 \mathrm{mM}$ glucose at low temperature and oxygen enrichment $\left(5 \% \mathrm{CO}_{2} / 95 \% \mathrm{O}_{2}\right)$. The $\mathrm{pH}$ value of the aCSF was adjusted to $7.3-7.4$.

\subsection{Experimental setup}

In this study, the indentation device was structured as described by Huang et al. ${ }^{[26]}$, as shown in Fig. 1a. The device was controlled by a user-defined LabVIEW programme (LabVIEW 2012, national instruments). Considering that the large deformation of this experiment far exceeded the sub-indentation depth threshold of traditional nano indentation, a servo motor (SGMJV-01ADA 61 , YASKAWA) was used to drive the alloy indenter.

\subsection{Test protocol}

\subsubsection{Indentation-relaxation test}

Based on the function of brain tissues, the cerebrum has been divided into four regions: Frontal Lobe (FL), Parietal Lobe (PL), Temporal Lobe (TL), and Occipital Lobe (OL). Fig. 1b displays these four regions and the location of each test point. To observe the maximum difference in mechanical properties in different regions clearly, indentation tests were performed at the locations shown by black dots in Fig. 1b; white dotted circles represented the error ranges of the test points. The results of this test did not include the PL region because when the sample was placed horizontally in the culture dish, no approximate plane existed on the PL surface area. Thus, it could not guarantee sufficient contact between the indenter and brain tissue (II in Fig. 1). In contrast, the other three areas (FL, TL, and OL) were in accordance with the test condition, and their test planes 

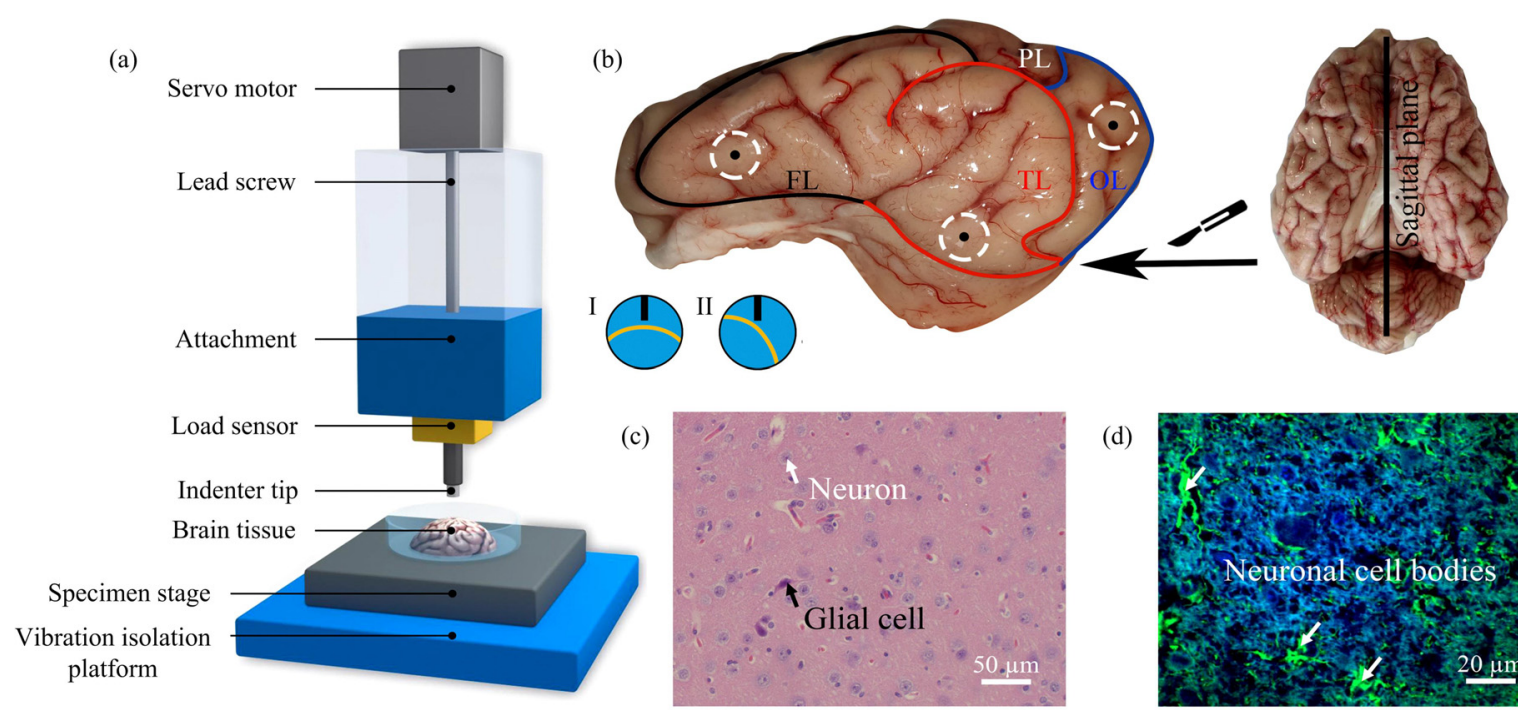

(c)

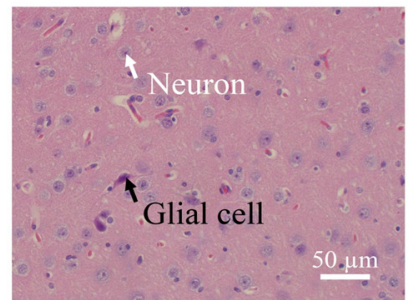

(d)

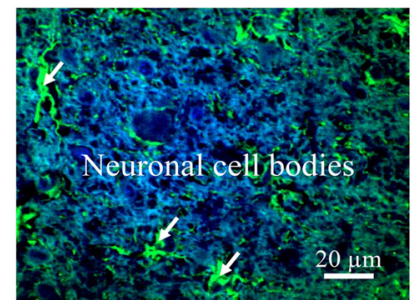

Fig. 1 Experimental equipment and samples. (a) Three-dimensional schematic diagram of experimental indentation instrument; (b) brain regions and sampling locations (the white dotted circle is the error range of the test point; black dots represent the projection directly below the indenter, and I and II represent the relative positions of the indenter and brain tissue for different regions); (c) a sample of $\mathrm{H} \& \mathrm{E}$ staining; (d) a sample of immunofluorescence staining.

were similar (I in Fig. 1); therefore, only these regions were included in this test. First, the whole brain tissue was excised into two complete half brain samples along the sagittal plane by using a scalpel, as shown in Fig. 1b. Further, a half brain sample was placed in a culture dish. During the test, the brain tissue was soaked in aCSF to avoid dehydration and adhesion. Simultaneously, the bottom of each sample was fixed with ethyl $\alpha$-cyanoacrylate to reduce the experimental error caused by the buoyancy of aCSF. In addition, to avoid dehydration caused by long-term exposure of the test surface to air, only one indentation test was conducted for each half brain sample. To establish the contact point with the tissue, the indenter tip was brought into close proximity with the tissue. Once the indenter was sufficiently close to the tissue, it was lowered in $2 \mu \mathrm{m}$ increments until a force of $<0.5 \mathrm{mN}$ was recorded from the indenter tip. If the force at any region increased to more than $0.5 \mathrm{mN}$ during the procedure, the region was not measured and the indenter was moved to a new location within the error range for testing. All the experiments were performed at room temperature and within $6 \mathrm{~h}$ of decapitation to reduce the influence of tissue proteolysis and necrosis on the accuracy of experimental results ${ }^{[12]}$. Notably, the mechanical properties of the left and right hemispheres do not differ significantly ${ }^{[27]}$. Consequently, the results of the left and right brain hemispheres were combined and not specifically distinguished during data processing.

Considering the small rigidity of brain tissue, a cylindrical aluminum alloy flat indenter with a radius of $1 \mathrm{~mm}$ was used. Since the purpose of this study was to measure the mechanical properties of brain tissues during the process of large deformation, the displacement of the indenter was $2000 \mu \mathrm{m}$. The average thickness of the indentation position measured by digital caliper was approximately $20 \mathrm{~mm}$, and the test scheme met the recommended boundary effect range of $10 \%$ (indentation depth/tissue height $)^{[28]}$. In previous studies, most of the indentation processes of biological tissues were characterised by force-time curves ${ }^{[29,30]}$. Fig. 2 a shows an average force-time curve of OL measured in this experiment (the observed trend for other curves was identical; therefore, they are not listed). The curve was composed of the approximate linear interval of the loading portion and the nonlinear interval of the relaxation portion. The elastic behaviour of brain tissues can be calculated by Sneddon's solution ${ }^{[31]}$, according to the slope of each force-depth curve (the force-depth mosaic in Fig. 2a) by using the Eq. (2) of section 2.4. Notably, the force-depth curve was not found to be completely linear. At the beginning of the curve, the collected data 

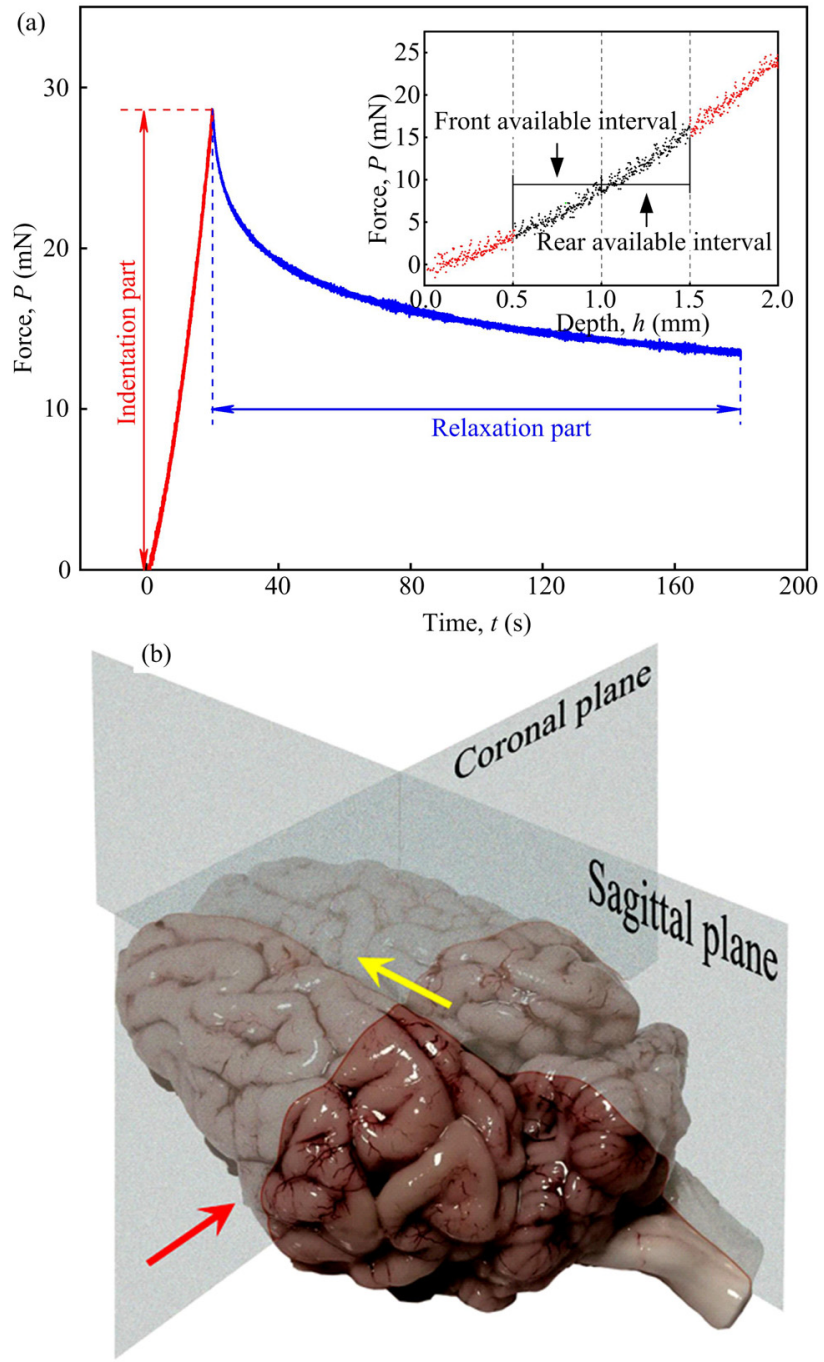

Fig. 2 (a) As an example in group \#2 of the test, the mosaic map is the original data of force-depth curve. (b) Direction of pathological section (yellow arrow) and direction of observation (red arrow).

represented obvious divergence phenomenon because of the adhesion of biological tissues. Before the end of the indentation process, the curve displayed obvious nonlinearity. The main reason for this phenomenon was the vibration of the servo motor, which caused the last curve of the loading section to drift. Therefore, to accurately characterise the elastic behaviour of cerebral cortex, $501 \mu \mathrm{m}-1500 \mu \mathrm{m}$ of the loading segment was used when the raw data was processed. However, a small wave of slope in the available data interval was still present due to the deep indentation depth and large strain. After analysing the characteristics of all the original data curves, we selected the midpoint as the cut-off point of slope change and calculated the shear modulus of the front
Table 1 Test parameters of each group

\begin{tabular}{|c|c|c|c|c|}
\hline Group & Test region & $\begin{array}{c}\text { Loading time } \\
(\mathrm{s})\end{array}$ & $\begin{array}{c}\text { Relaxation } \\
\text { time }(\mathrm{s})\end{array}$ & $\begin{array}{c}\text { Loading speed } \\
\left(\mu \mathrm{m} \cdot \mathrm{s}^{-1}\right)\end{array}$ \\
\hline$\# 1$ & FL, TL, OL & 4 & 176 & 500 \\
\hline$\# 2$ & FL, TL, OL & 20 & 160 & 100 \\
\hline$\# 3$ & FL, TL, OL & 40 & 140 & 50 \\
\hline
\end{tabular}

half and rear half to characterise the mechanical properties of the brain tissue more accurately under large strain.

To ensure the accuracy of the test results, study on three groups, including one group with fast-loading mode (with a loading time of $4 \mathrm{~s}$ ) and two groups with slow-loading mode (with loading times of $20 \mathrm{~s}$ and $40 \mathrm{~s}$ ), of samples was conducted on the same area. The times of measurement points in each area of groups $\# 1-\# 3$ were 6 , and the total times of measurement points in this test were 54. Table 1 presents the detailed test parameters of each group.

\subsubsection{Histological characterisation}

After completion of the indentation experiment of each sample of group \#1, the brain tissue was excisedusing a scalpel at the indentation point, followed by fixation ( $10 \%$ formalin solution), dehydration ( $90 \%$ ethanol solution), paraffin-embedding, staining, and preparation of pathological sections. Two types of histological staining were performed. The nucleus was visualised in blue (Fig. 1c) in H \& E staining. After immunofluorescence staining, the neurons appeared green (Fig. 1d) under a laser confocal microscope. The direction of pathological section was parallel to the sagittal plane, as shown in Fig. 2b. For quantifying the microscopic components, we randomly selected a $5 \times 10^{4} \mu \mathrm{m}^{2}$ area on each slice as the effective unit area under 120-times magnification, counted the number of cells in the unit area, and considered the number of cells in the effective unit area as the only quantitative index.

\subsection{Theory}

In this paper, the Sneddon's solution ${ }^{[31]}$ was used to calculate the shear modulus during the indentationpart:

$$
P=\frac{4 \kappa R h G}{1-v} .
$$

Qian et al. ${ }^{[30]}$ further simplified Sneddon's solution, leading to Eq. (2): 


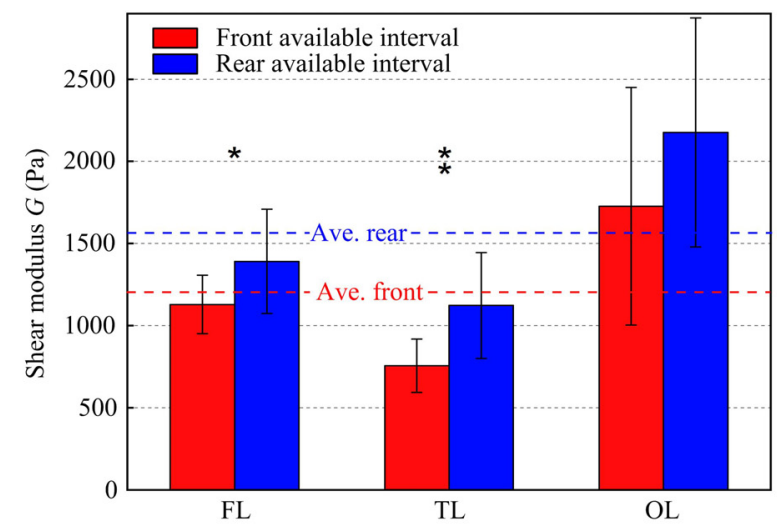

(a)

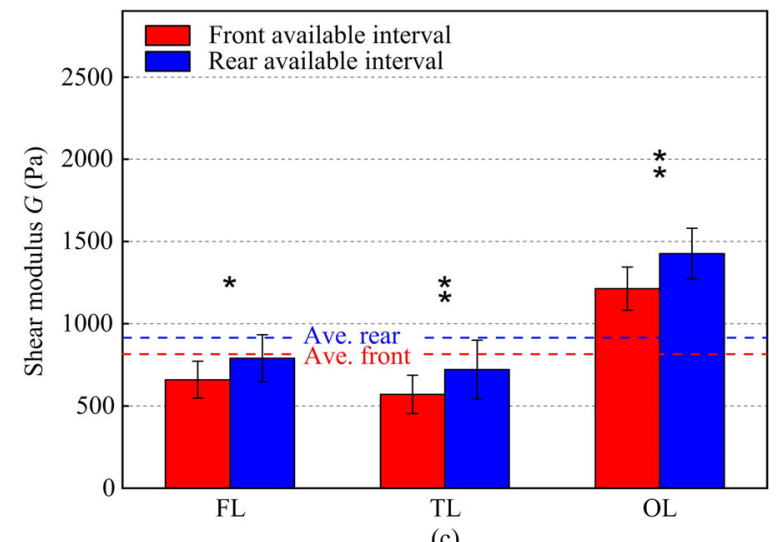

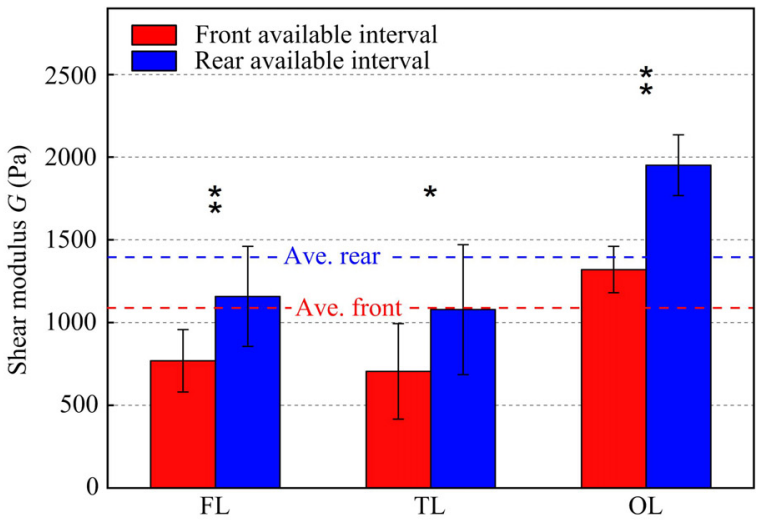

(b)

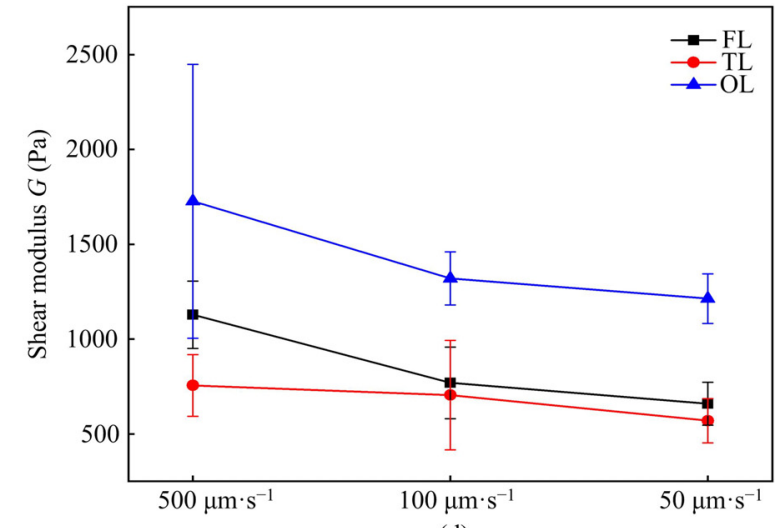

(d)

Fig. 3 The shear modulus of the brain tissue with indentation speeds of (a) $500 \mu \mathrm{m} \cdot \mathrm{s}^{-1}$, (b) $100 \mu \mathrm{m} \cdot \mathrm{s}^{-1}$, and (c) $50 \mu \mathrm{m} \cdot \mathrm{s}^{-1}$. The front- and rear-half moduli were calculated using Bonferroni-corrected post-hoc tests $\left({ }^{*} p<0.05,{ }^{* *} p<0.001\right)$. (d) The change in modulus with indentation speed of different test regions (front available interval).

$$
G=\frac{1-v}{4 \kappa R} \cdot \frac{\Delta P}{\Delta h},
$$

where $P$ represents the instantaneous contact force, $R$ represents the radius of the cylindrical flat indenter, $h$ represents the indentation depth, $G$ represents the shear modulus, $v$ represents the Poisson's ratio (assumed to be $0.5)$, and $\kappa$ represents the correction factor.

A Boltzmann genetic integral ${ }^{[32]}$ can be used to characterize the shear modulus of the whole indentation-relaxation curves:

$$
P(t)=\frac{4 \kappa R}{1-v} \int_{0}^{t} G(t-\tau)\left(\frac{\mathrm{d} t}{\mathrm{~d} \tau}\right) .
$$

Prony series approximation can be used to express $G(t)$ in Eq. (3):

$$
G(t)=G_{\infty}+\sum_{j} G_{j} \cdot \exp \left(-t / \tau_{j}\right),
$$

where $G_{\infty}$ represents the equilibrium shear modulus, $\tau_{j}$ represents the time constant of each relaxation process, and $G_{j}$ represents the corresponding shear modulus of each exponential term. In MATLAB (R 2016 b), lsqcurvefit. $\mathrm{m}$ and nlinfit. $\mathrm{m}$ functions were used to fit the original data, from which the change curve of the shear modulus over the whole process was obtained. Hayes et al. showed that the coefficient is related to the value of the radius of the indenter, the total thickness of the tested sample, and Poisson's ratio, thus obtaining the calculation formula for $\kappa^{[33]}$. In this study, a cylindrical flat indenter with a radius of $1 \mathrm{~mm}$ was used. The average thickness of the sample was $20 \mathrm{~mm}$, and the final $\kappa$ value was 1.06 .

\section{Results}

\subsection{Elastic behaviour of the cerebral cortex}

In terms of shear modulus, the laws shown in Figs. $3 a-3 c$ were found to be consistent, that is, the test points on the $\mathrm{OL}$ and TL regions had the highest and 
lowest moduli, respectively, which shows that the cerebral cortex in the TL region is the softest among the three test regions. By observing the difference in modulus between the front available interval and rear available interval, we found that the average modulus of the front interval is lower than that of the rear interval; however, the difference was gradually disappeared with decrease in the indentation speed. Fig. 3d depicts the sensitivity of the three test regions to changes in the loading speed. When the indentation speed dropped from $500 \mu \mathrm{m} \cdot \mathrm{s}^{-1}$ to $50 \mu \mathrm{m} \cdot \mathrm{s}^{-1}$, the value of shear modulus of the TL changed to a small extent, and the average shear modulus only dropped from $755.59 \mathrm{~Pa} \pm 162.45 \mathrm{~Pa}$ to $569.85 \mathrm{~Pa} \pm$ 116.53 Pa. However, under similar conditions, the value of shear modulus of the OL region decreased from $1726.79 \mathrm{~Pa} \pm 722.89 \mathrm{~Pa}$ to $1213.38 \mathrm{~Pa} \pm 130.46 \mathrm{~Pa}$. These results indicated that the local mechanical properties of the brain tissue are easily affected by the loading speed. The OL region was found to be more sensitive to changes in the loading speed, whereas the TL region exhibited a more compliant response to changes in the loading speed. The mechanical properties of the TL region were found to be more stable than those of the $\mathrm{OL}$ and FL regions. In addition, the length of the error bars was compared; which indicated that for the same region, the smaller is the indentation speed, the more compact are the obtained data points and the shorter is the error bar. The finding confirms that rapid loading can significantly eliminate the phenomenon of creep and reflect individual differences in brain tissues. Notably, the variation in test results due to different sampling individuals was found to be inevitable. In this study, the method of averaging multiple measurements was used to minimise the error.

\subsection{Relaxation behaviour of cerebral cortex}

Figs. $4 \mathrm{a}-4 \mathrm{c}$ show the relationship between the stress and relaxation time. The relaxation curves of the three test regions were found to be similar. The main manifestation was that the stress decreased rapidly and reached a pseudo-equilibrium. These curves can describe the stress relaxation behaviour of a typical viscoelastic model. From the peak force at the beginning of the relaxation part, the test points on the OL region had the highest initial stress, which corresponded to the re- sults of this paper for the shear modulus of the three test points. To contrast the time-dependent characteristics of the three regions, Eq. (3) was used to fit the relevant parameters (because the other two groups had the same law, only the first group in Table 2 is taken as an example), and further, the parameters were substituted into Eq. (4) to draw the normalised shear modulus curve with time (as shown in Figs. $4 \mathrm{~d}-4 \mathrm{f}$ ). In addition, the average relaxation rates after $140 \mathrm{~s}$ were calculated according to the original data, and the results are shown in the mosaic diagram of Figs. $4 d-4 f$. For the time constants in Table 2, the first constant $\tau_{1}$ represents the response speed of brain tissue to stress changes; the response speed of TL region was found to be significantly slow, which corresponded to the insensitivity of the TL region to speed changes in the previous section. In addition, it can be seen that from the beginning of relaxation part to pseudo-equilibrium, the shear modulus of the FL region decreased significantly, indicating that compared with other regions, the viscosity of the FL region of the cerebral cortex was greater. Moreover, the FL region of the cerebral cortex responded more rapidly than the $\mathrm{OL}$ and TL regions. At a loading speed of $500 \mu \mathrm{m} \cdot \mathrm{s}^{-1}$ (Fig. 4d), the shear modulus of the FL region decreased approximately by $40 \%$ at $20 \mathrm{~s}$ after the beginning of relaxation, which was approximately $10 \%-15 \%$ faster than that of the other two regions. Comparison between the stress relaxation rate of each test point in $140 \mathrm{~s}$ after the indentation indicated that the stress relaxation rate of the test point on the FL region is the highest. In addition, as the indentation speed decreased, the stress relaxation rate of the test points in the same area reduced.

\subsection{Correlation analysis between microstructure and mechanical properties}

We compared the total number of nuclei and the number of neuronal cell bodies at three test points per unit area after quantification, and the results are expressed in the form of a histogram in Figs. 5a and 5b. As shown in Fig. 5a, the density of neuronal cell body was the highest at the OL test point. Thus, the neural network in the back of the brain was dense. As shown in Fig. 5b, the density of total nuclei at the TL test point of the cerebral cortex was found to be the largest, whereas the density of cell nuclei at the OL test point was found to be 


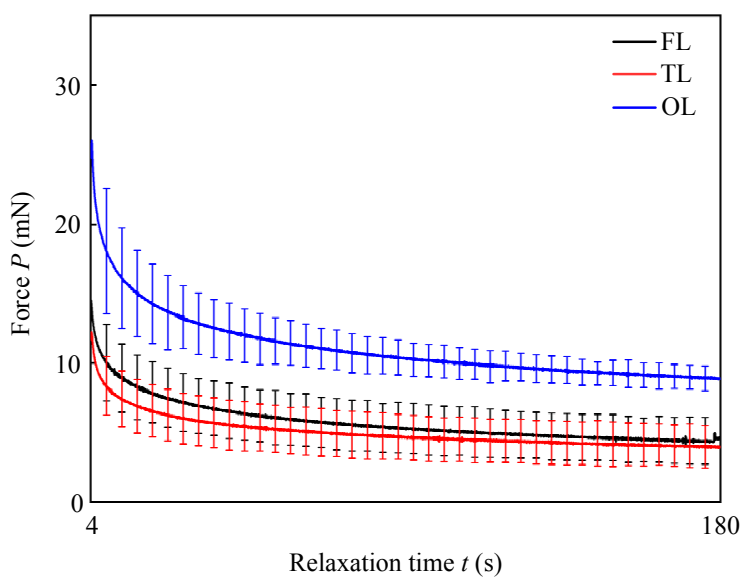

(a)

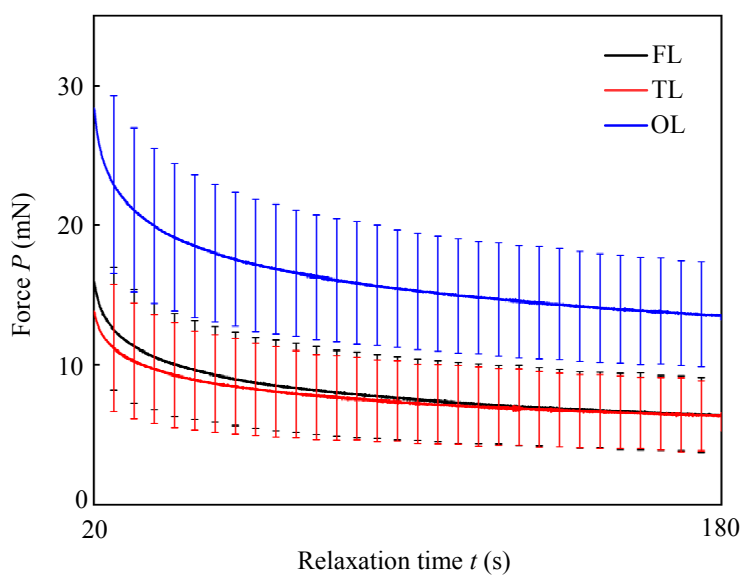

(b)

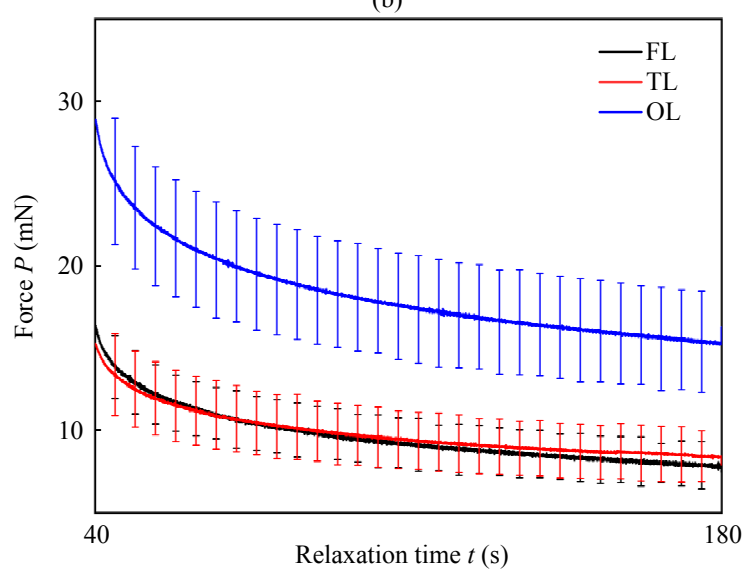

(c)

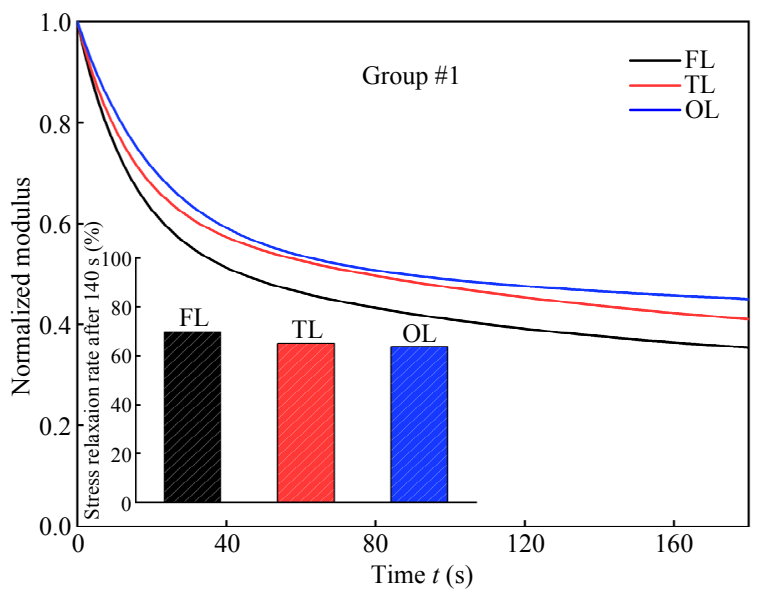

(d)

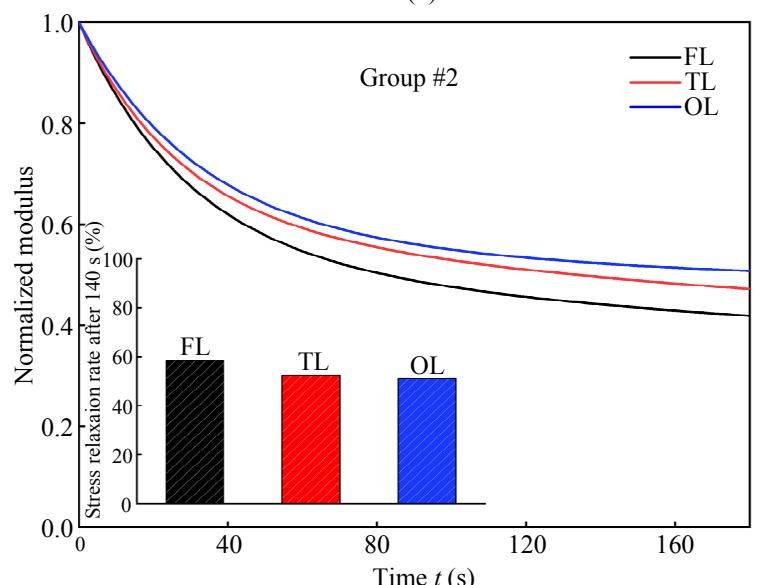

(e)

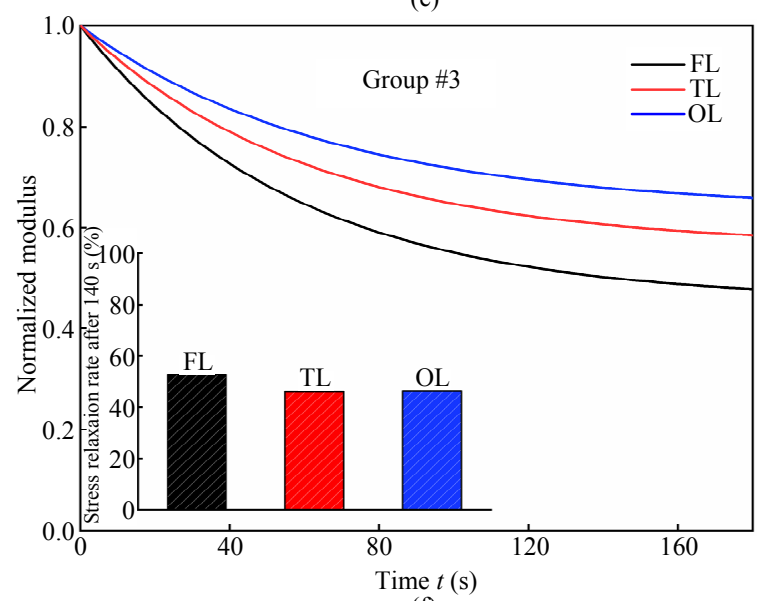

(f)

Fig. 4 Relaxation curves of brain tissue with indentation speeds of (a) $500 \mu \mathrm{m} \cdot \mathrm{s}^{-1}$, (b) $100 \mu \mathrm{m} \cdot \mathrm{s}^{-1}$, and (c) $50 \mu \mathrm{m} \cdot \mathrm{s}^{-1}$. (d) - (f) Changes in the shear modulus and relaxation rate after $140 \mathrm{~s}$ in groups $\# 1-\# 3$.

Table 2 Prony series parameters with 95\% confidence intervals for group \#1 of different regions

\begin{tabular}{ccccccc}
\hline$G_{j}(\mathrm{~Pa}), \tau_{j}(\mathrm{~s})$ & $G_{0}$ & $G_{1}$ & $\tau_{1}$ & $G_{2}$ & $\tau_{2}$ \\
\hline $\mathrm{FL}$ & $222.25 \pm 6.07$ & $293.79 \pm 13.17$ & $12.50 \pm 0.70$ & $242.33 \pm 4.41$ & $106.64 \pm 7.12$ & 0.97 \\
$\mathrm{TL}$ & $189.58 \pm 10.85$ & $213.53 \pm 5.76$ & $14.32 \pm 0.59$ & $167.38 \pm 5.50$ & $121.31 \pm 8.26$ & 0.96 \\
$\mathrm{OL}$ & $444.65 \pm 16.30$ & $449.23 \pm 20.55$ & $11.24 \pm 0.41$ & $362.35 \pm 6.98$ & $96.79 \pm 10.87$ & 0.96 \\
\hline
\end{tabular}




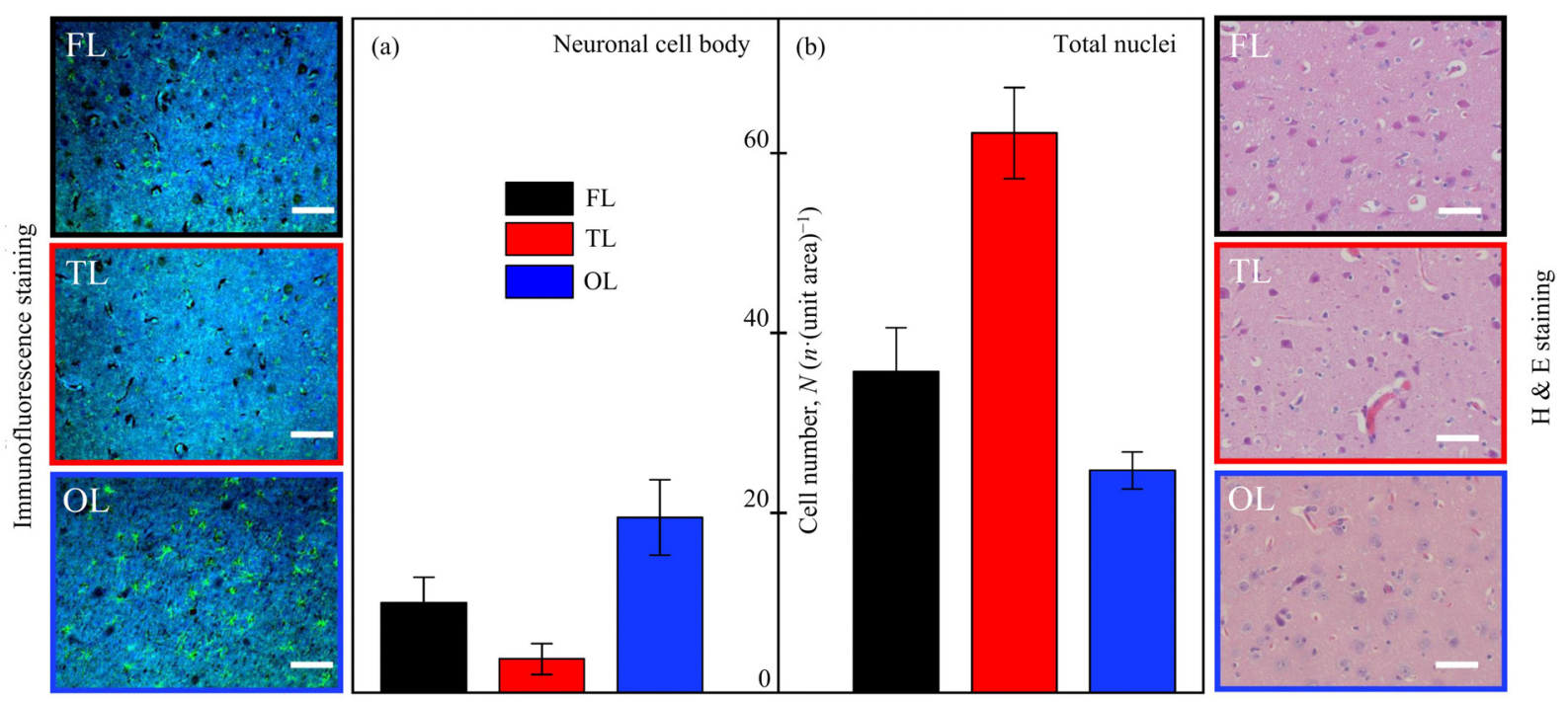

Fig. 5 Density of neuron (a) and total cell nucleus (b) quantified through immunofluorescence staining and H \& E staining (group \#1). The unit area is $5 \times 10^{4} \mu \mathrm{m}^{2}$ (scale bar $\left.=50 \mu \mathrm{m}\right)$.
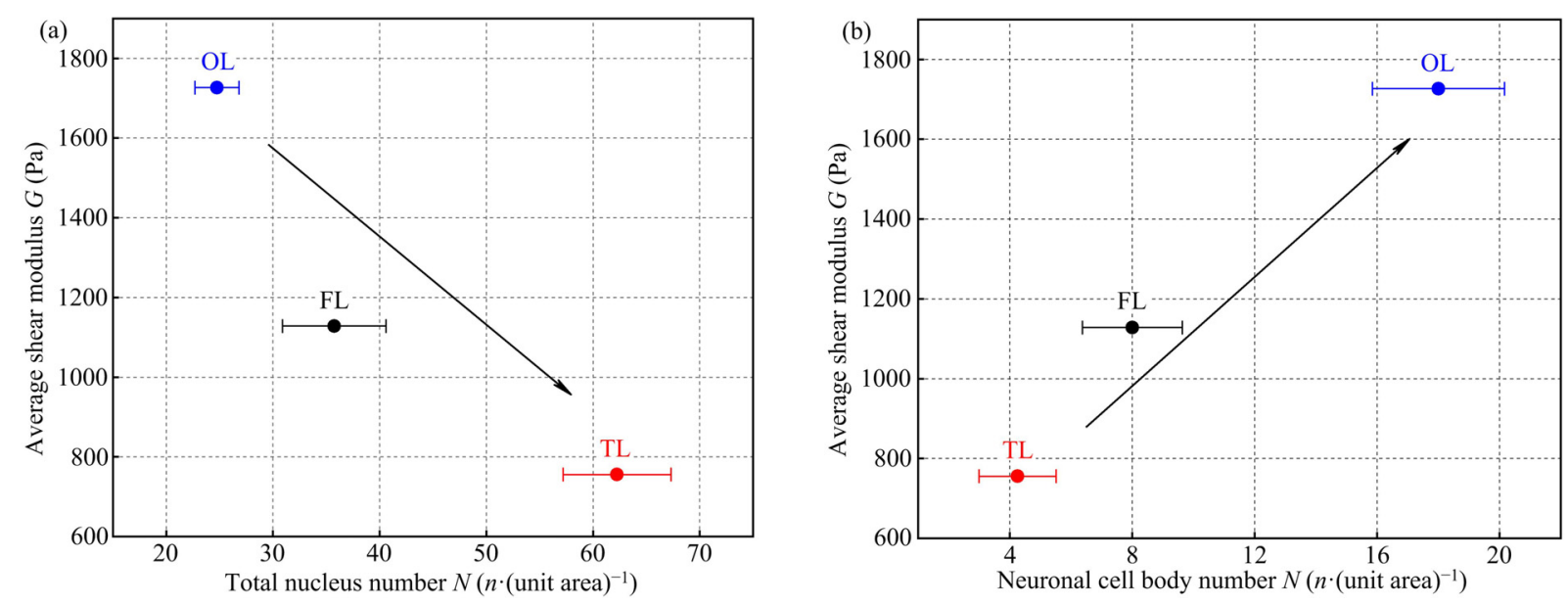

Fig. 6 (a) Correlation between mechanics and total nuclei (mean \pm standard deviation). (b) Correlation between mechanics and neuronal cell body (mean \pm standard deviation). The unit area was $5 \times 10^{4} \mu \mathrm{m}^{2}$.

the least. We can infer that the cell distribution varies across different areas of the cerebral cortex.

Fig. 6a illustrates the correlation between the shear modulus of the three test points and the total number of cells. For the cerebral cortex, the shear modulus of the local area was found to be negatively correlated with the cell density, which implies that the more is the total number of cells per unit area, the softer is the brain tissue. However, the shear modulus of the brain tissue was found to be positively correlated with the neuronal density (Fig. 6b), which implies that the relative hardness of the brain tissue is the greatest in the regions where the neurons are densely distributed.

\section{Discussion}

In recent years, the mechanical properties of brain tissue have attracted much attention, and great efforts have been made to study these properties. However, combining the mechanical differences of brain tissues with the distinctions of their microstructure and explaining the mechanism underlying the differences in the mechanical properties of brain tissues till remain challenging. In this study, the indentation method was used to test the mechanical properties of the porcine brain in different cerebral cortex regions. $\mathrm{H} \& \mathrm{E}$ and immunofluorescence staining methods were used to count the number of cells per unit area, and finally, the correlation 
between the mechanical properties of biological tissues and density of cells was studied.

In the indentation portion, the porcine cerebral cortex exhibited distinct elastic behaviour, which is consistent with the findings of previous studies. For example, Gefen et al. ${ }^{[34]}$ measured the shear modulus of grey matter in the porcine cortex in vitro as $G=1.3 \mathrm{kPa}$, and Elkin et al. ${ }^{[10]}$ measured the shear modulus of grey matter in the cortex of adult rats as $G=2.7 \mathrm{kPa}$. However, several previously published conclusions are inconsistent with the data of this study. Kaster et al. ${ }^{[35]}$ reported that the shear modulus of grey matter is $0.4 \mathrm{kPa}$, which was obtained through the indentation of the porcine brain tissue, and Dummelen et al. ${ }^{[25]}$ reported that the shear modulus of grey matter is $0.7 \mathrm{kPa}$. These moduli are clearly smaller than that observed in the current study. This is likely because of different test drafts, test environments, and other factors. In addition, this study revealed the effect of cell number on local shear modulus. For biomaterials with complex structure, such as cerebral cortex, the individual differences in organisms and the selective expression of genes in specific regions will lead to variation in test results between different samples, which increases the length of error bar in the independent repeated experiment. However, in the present study, all the original data were statistically tested during the process of data. Moreover, the three groups of experimental data followed the same rule, which is enough to prove the accuracy of the experimental data in this study.

Figs. $4 \mathrm{~d}-4 \mathrm{f}$ show that at the same test region, with the extension of the indentation time (from $4 \mathrm{~s}$ of group \#1 to $20 \mathrm{~s}$ of group \#3), the relaxation rate gradually decreased after $140 \mathrm{~s}$ of the start of the relaxation part. This proves that the stress relaxation phenomenon exists in the whole indentation-relaxation process. In previous reports ${ }^{[30]}$, this phenomenon has been explained only as an empirical rule. Here, we proved that the stress relaxation behaviour of the cerebral cortex is transient. The cerebral cortex of the three test points tested in this study showed different time-dependent characteristics during the indentation-relaxation process, which may be related to the uneven cell distribution in the local region. Simultaneously, the permeability will also affect the viscous behaviour of the brain tissue ${ }^{[20]}$, which will be explored in further research.

According to our analysis, the shear modulus of the cerebral cortex is negatively correlated with the total number of cells per unit area, that is, the regions where the cells are densely distributed, the brain tissue is soft. This conclusion is consistent with that of previous studies ${ }^{[36]}$. The interaction force between the phospholipid molecules that constitute the cell membrane is weak, and it is prone to dislocation, distortion, and stretching. Therefore, cells are the softest components in the brain tissue, and hence, they have little impact on mechanical properties. The finding also explains the insensitivity of the TL test area to load changes. In addition, we used immunofluorescence staining to count neuronal cell bodies, and the number of neurons per unit area of the cerebral cortex was positively correlated with the shear modulus; that is, the brain tissue was relatively hard at the regions where the neurons were densely distributed. According to a previous study ${ }^{[37]}$, the cell types in the cerebral cortex can be divided into pyramidal neurons and glial cells. The glial cells can be further divided into protoplasmic astrocytes, oligodendrocytes, and microglial cells. Because of the limitation of the staining method used in this study, the glial cells could not be distinguished and quantified in detail. However, the ratio of the total number of cells to the neuronal cell bodies measured at each test point provided an indirect estimation of the ratio of glial cells (Fig. 7). Glial cells accounted for the largest proportion of the TL test point and the smallest proportion of the OL test point. Previous studies have indicated that glial cells do not serve as rigid cement-like structural supports for the neuronal network; rather, they act as a soft viscoelastic padding (shock absorbers) for neurons. Thus, they prevent or at least minimise neuronal damage in case of mechanical trauma ${ }^{[38]}$. This observation provides a reasonable explanation for the compliance of TL test points to changes in the loading speed. In terms of the proportion of neurons, the proportion of neurons was higher at the OL test point compared with that at other two test points. According to previous reports, the stiffness of a single glial cell is less than that of a neuron ${ }^{[39]}$, which also explains the relatively high shear modulus at the OL test point. For a complex anisotropic material, such as brain tissue, combining the microstructure with mechanical properties 


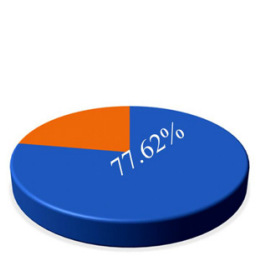

FL

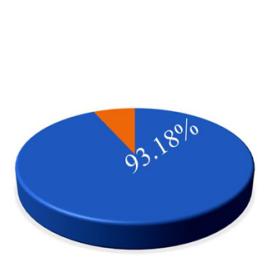

TL

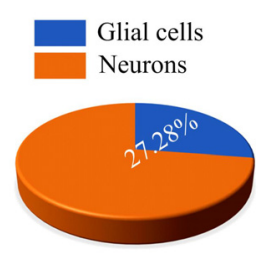

$\mathrm{OL}$
Fig. 7 Average ratio of glial cells to neurons at each test point.

and exploring the mechanism are still challenging. According to previous studies, not only the cells but also the biological macromolecules (phospholipids ${ }^{[40]}$, proteoglycans ${ }^{[41]}$, and collagen ${ }^{[20]}$ ) seem to have more significant effects on the mechanical properties of brain tissues. Therefore, in future studies, we will explore the impact of biomacromolecule content on the mechanical properties of brain tissues.

\section{Conclusion}

In this study, we have elucidated the regional mechanical properties of brain tissues based on the density of total nuclei and neuronal cell bodies. We are the first to carry out mechanical performance tests according to brain functional divisions, and explore the correlation between the neuronal cell bodies and the mechanical properties in the cerebral cortex. We found that in the porcine cerebral cortex, the FL had the strongest viscosity, the TL had the lowest sensitivity to changes in indentation speed, and the OL had the highest shear modulus. In addition, our study reveals a negative correlation between the total cell count and the stiffness, a positive correlation between neuronal cell body count and stiffness. Understanding the region-specific mechanical behavior of brain tissues based on the cell density will help us design more accurate, multi-scale finite element analysis models of the brain. This is a critical step towards a better understanding of the role of mechanics in clinical conditions including chronic traumatic encephalopathy, cerebral ischemic disease, or neurodegenerative diseases.

\section{Acknowledgment}

This work was supported by the National Key R\&D Program of China (2018YFF01012400), Jilin Provincial Middle and Young Scientific and Technological Innovation Talent and Team Project (20170519001JH), and Program for JLU Science and Technology Innovative
Research Team (2017TD—04).

Open Access This article is licensed under a Creative Commons Attribution 4.0 International License, which permits use, sharing, adaptation, distribution and reproduction in any medium or format, as long as you give appropriate credit to the original author(s) and the source, provide a link to the Creative Commons licence, and indicate if changes were made.

The images or other third party material in this article are included in the article's Creative Commons licence, unless indicated otherwise in a credit line to the material. If material is not included in the article's Creative Commons licence and your intended use is not permitted by statutory regulation or exceeds the permitted use, you will need to obtain permission directly from the copyright holder.

To view a copy of this licence, visit http://creativecommons.org/licenses/by/4.0/.

\section{References}

[1] Jeremy A, Misaal P, Dylan F, Xin A, Catherine C, Quinn W, Rebecca R, Li C. A novel mouse model for the study of endogenous neural stem and progenitor cells after traumatic brain injury. Experimental Neurology, 2020, 325, 113119.

[2] Song J, Lee S-S, Lim S, Yeo S. Mechanism of the neuroprotective effect of injecting brain cells on ST36 in an animal model of Parkinson's disease. Neuroscience Letters, 2020, 717, 134698 .

[3] Kansal A R, Torquato S, Harsh G R, Chiocca E A, Deisboeck T S. Simulated brain tumor growth dynamics using a three-dimensional cellular automaton. Journal of Theoretical Biology, 2000, 203, 367-382.

[4] Cloots R, van Dommelen J, Kleiven S, Geers M. Multi-scale mechanics of traumatic brain injury: Predicting axonal strains from head loads. Biomechanics \& Modeling in Mechanobiology, 2013, 12, 137-150.

[5] Jin X, Zhu F, Mao H, Shen M, Yang K H. A comprehensive experimental study on material properties of human brain tissue. Journal of Biomechanics, 2013, 46, 2795-2801.

[6] Li Z, Ji C, Li D P, Luo R T, Wang G L, Jiang J Z. A comprehensive study on the mechanical properties of different regions of 8-week-old pediatric porcine brain under tension, shear, and compression at various strain rates. Journal of Biomechanics, 2020, 98, 109380.

[7] Haslach H W, Leahy L N, Riley P, Gullapalli R, Xu S, Hsieh A H. Solid-extracellular fluid interaction and damage in the 
mechanical response of rat brain tissue under confined compression. Journal of the Mechanical Behavior of Biomedical Materials, 2014, 29, 138-150.

[8] MacManus D B, Murphy J G, Gilchrist M D. Mechanical characterisation of brain tissue up to $35 \%$ strain at 1,10 , and 100/s using a custom-built micro-indentation apparatus. Journal of the Mechanical Behavior of Biomedical Materials, 2018, 87, 256-266.

[9] MacManus D B, Pierrat B, Murphy J G, Gilchrist M D. A viscoelastic analysis of the P56 mouse brain under large-deformation dynamic indentation. Acta Biomaterialia, 2017, 48, 309-318.

[10] Elkin B S, Morrison B. Viscoelastic Properties of the P17 and adult rat brain from indentation in the coronal plane. Journal of Biomechanical Engineering, 2013, 135, 114507.

[11] Gefen A, Gefen N, Zhu Q, Raghupathi R, Margulies S. Age-dependent changes in material properties of the brain and braincase of the rat. Journal of Neurotrauma, 2003, 20, 1163-1177.

[12] Budday S, Nay R, de Rooij R, Steinmann P, Wyrobek T, Ovaert T C, Kuhl E. Mechanical properties of gray and white matter brain tissue by indentation. Journal of the Mechanical Behavior of Biomedical Materials, 2015, 46, 318-330.

[13] Christ A F, Franze K, Gautier H, Moshayedi P, Fawcett J, Franklin R J M, Karadottir R T, Guck J. Mechanical difference between white and gray matter in the rat cerebellum measured by scanning force microscopy. Journal of Biomechanics, 2010, 43, 2986-2992.

[14] Menichetti A, Macmanus D B, Gilchrist M D, Depreitere B, Famaey N. Regional characterization of the dynamic mechanical properties of human brain tissue by microindentation. International Journal of Engineering Science, 2020, $\mathbf{1 5 5}, 103355$.

[15] MacManus D B, Pierrat B, Murphy J G, Gilchrist M D. Dynamic mechanical properties of murine brain tissue using micro-indentation. Journal of Biomechanics, 2015, 48, 3213-3218.

[16] Prevost T P, Jin G, de Moya M A, Alam H B, Suresh S, Socrate S. Dynamic mechanical response of brain tissue in indentation in vivo, in situ and in vitro. Acta Biomaterialia, 2011, 7, 4090-4101.

[17] Blumenthal N R, Hermanson O, HeimrichB, Shastri V P. Stochastic nanoroughness modulates neuron-astrocyte interactions and function via mechanosensing cation channels. Proceedings of the National Academy of Sciences of the United States of America, 2014, 111, 16124-16129.

[18] Tyler W J. The mechanobiology of brain function. Nature
Reviews Neuroscience, 2012, 13, 867-878.

[19] Bayly P V, Taber L A, Kroenke C D. Mechanical forces in cerebral cortical folding: A review of measurements and models. Journal of the Mechanical Behavior of Biomedical Materials, 2014, 29, 568-581.

[20] Budday S, Sarem M, Starck L, Sommer G, Pfefferle J, Phunchago N, Kuhl E, Paulsen F, Steinmann P, Shastri V P. Towards microstructure-informed material models for human brain tissue. Acta Biomaterialia, 2020, 104, 53-65.

[21] Sauleau P, Lapouble E, Val-Laillet D, Malbert C H. The pig model in brain imaging and neurosurgery. Animal, 2009, 3, $1138-1151$.

[22] Holm I E, Alstrup A K O, Luo Y. Genetically modified pig models for neurodegenerative disorders. Journal of $\mathrm{Pa}$ thology, 2016, 238, 267-287.

[23] Eaton S L, Wishart T M. Bridging the gap: Large animal models in neurodegenerative research. Mammalian Genome, 2017, 28, 324-337.

[24] MacManus D B, Menichetti A, Depreitere B, Famaey N, Vander Sloten J, Gilchrist M. Towards animal surrogates for characterising large strain dynamic mechanical properties of human brain tissue. Brain Multiphysics, 2020, 1, 100018

[25] Dommelen J A, Sande T P, Hrapko M, Peters G W. Mechanical properties of brain tissue by indentation: Interregional variation. Journal of the Mechanical Behavior of Biomedical Materials, 2010, 3, 158-166.

[26] Huang H, Zhao H W, Shi C L, Zhang L, Wan S G, Geng C Y. Randomness and statistical laws of indentation-induced pop-out in single crystal silicon. Materials (Basel), 2013, 6, 1496-1505.

[27] Jiang Y, Li G, Qian L X, Liang S, Destrade M, Cao Y J. Measuring the linear and nonlinear elastic properties of brain tissue with shear waves and inverse analysis. Biomechanics \& Modeling in Mechanobiology, 2015, 14, 1119-1128.

[28] Garo A, Hrapko M, Dommelen J A, Peters G W. Towards a reliable characterisation of the mechanical behaviour of brain tissue: The effects of post-mortem time and sample preparation. Biorheology, 2007, 44, 51-58.

[29] Qian L, Sun Y F, Tian J Y, Ren Z, Zhao H W. Indentation response in porcine brain under electric fields. Soft Matter, 2019, 15, 623-632.

[30] Qian L, Zhao H W, Guo Y, Li Y, Zhou M, Yang L, Wang Z, Sun Y F. Influence of strain rate on indentation response of porcine brain. Journal of the Mechanical Behavior of Biomedical Materials, 2018, 82, 210-217.

[31] Sneddon. The relation between load and penetration in the axisymmetric boussinesq problem for a punch of arbitrary 
profile. International Journal of Engineering Science, 1965, 3, 47-57.

[32] Lee E H, Radok J. The contact problem for viscoelastic bodies. Journal of Applied Mechanics, 1960, 27, 364-395.

[33] Hayes W C, Keer L M, Herrmann G, Mockros L F. A mathematical analysis for indentation tests of articular cartilage. Journal of Biomechanics, 1972, 5, 541-551.

[34] Gefen A, Margulies S S. Are in vivo and in situ brain tissues mechanically similar. Journal of Biomechanics, 2004, 37, 339-1352.

[35] Kaster T, Sack I, Samani A. Measurement of the hyperelastic properties of ex vivo brain tissue slices. Journal of Biomechanics, 2011, 44, 1158-1163.

[36] Antonovaite N, Beekmans S V, Hol E M, Wadman W J, Iannuzzi D: Regional variations in stiffness in live mouse brain tissue determined by depth-controlled indentation mapping. Scientific Reports, 2018, 8, 12517.
[37] Ge W P, Jia J M. Local production of astrocytes in the cerebral cortex. Neuroscience, 2016, 323, 3-9.

[38] Cordiglieri C, Farina C. Astrocytes exert and control immune responses in the brain. Current Immunology Reviews, 2010, 6, 150-159.

[39] Lu Y B, Franze K, Seifert G, Steinhäuser C, Kirchhoff F, Wolburg H, Guck J, Janmey P, Wei E Q, Käs J, Reichenbach A. Viscoelastic properties of individual glial cells and neurons in the CNS. Proceedings of the National Academy of Sciences, 2006, 103, 17759-17764.

[40] Weickenmeier J, de Rooij R, Budday S, Steinmann P, Ovaert T C, Kuhl E. Brain stiffness increases with myelin content. Acta Biomaterialia, 2016, 42, 265-272.

[41] Mahmood M F, Clarke M J, Riches D P. Proteoglycans exert a significant effect on human meniscal stiffness through ionic effects. Clinical Biomechanics, 2020, 77, 105028. 